\title{
Arthroskopisch gestützte Reposition von posterolateralen Kantenfragmenten am Tibiakopf mit einem angeschrägten, kanülierten Repositionsstößel
}

\author{
Carsten Schlickewei, Karl-Heinz Frosch
}

Posterolaterale Kantenfragmente am Tibiakopf ( $\vee$ Abb. 1) werden in ihrer klinischen Bedeutung oft unterschätzt! Die offene Reposition und Refixation ist aufwendig und erfordert spezialisierte posterolaterale Zugänge [1]. Häufig führen schlechte Repositionen oder sekundär dislozierte Fragmente zu funktionell schlechten Ergebnissen [2-4]. Insbesondere kann eine knöchern bedingte rotatorische Instabilität im Sinne eines rezidivierenden Pivot-Shift-Phänomens verbleiben.

Eine einfachere Methode zur operativen Versorgung posterolateraler Kantenfragmente ist die arthroskopisch gestützte Reposition des Fragments mit einem kanülierten, angeschrägten Stößel und die anschließende perkutane Schraubenosteosynthese.

Nach einer diagnostischen Arthroskopie wird die Fraktur mit dem Bildwandler lokalisiert. Arthroskopisch gestützt wird ein Zieldraht mithilfe eines Kreuzbandzielbügels in Richtung des Kantenfragments vorgebohrt (• Abb. 2). Unter radiologischer und arthroskopischer Kontrolle wird der kanülierte Stößel (Arthrex, Naples, USA) zur vorsichtigen Reposition des Fragments genutzt ( $\triangleright$ Abb. 3 ). Nach anatomischer Reposition des Fragments kann die Fraktur

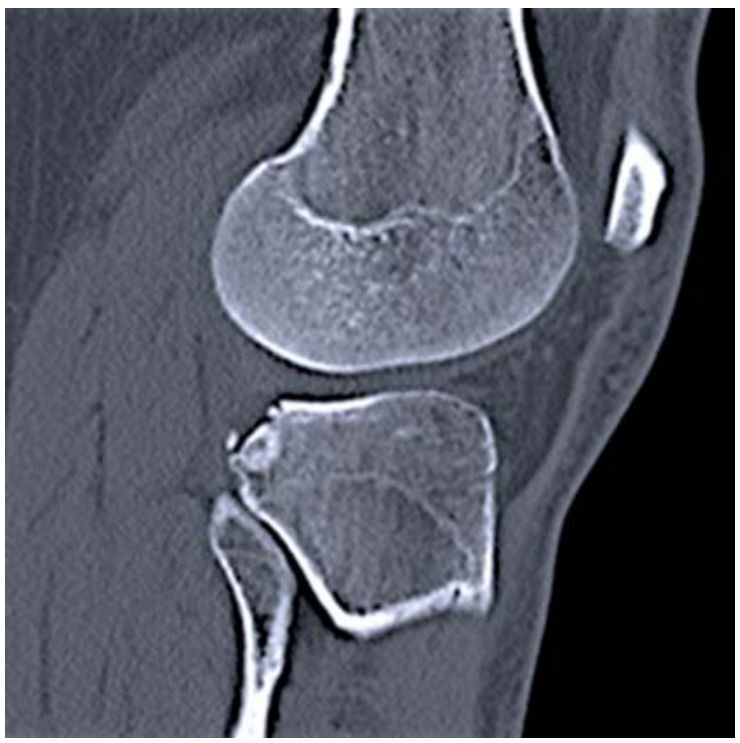

- Abb. 1 Posterolaterales Kantenfragment.

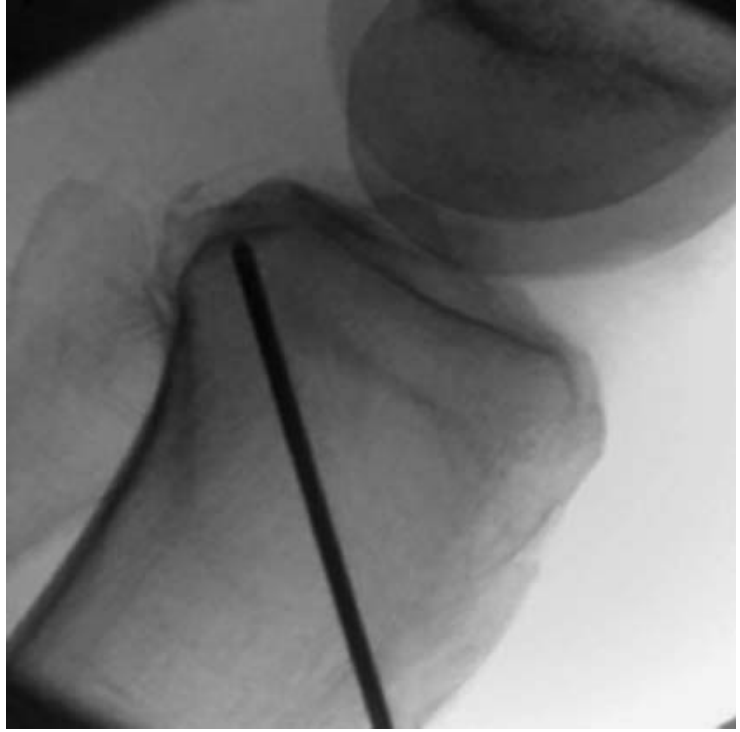

- Abb. 2 Adressierung der Fraktur mit Hilfe eines Zieldrahtes, über welchen der angeschrägte Stößel eingebracht werden kann.

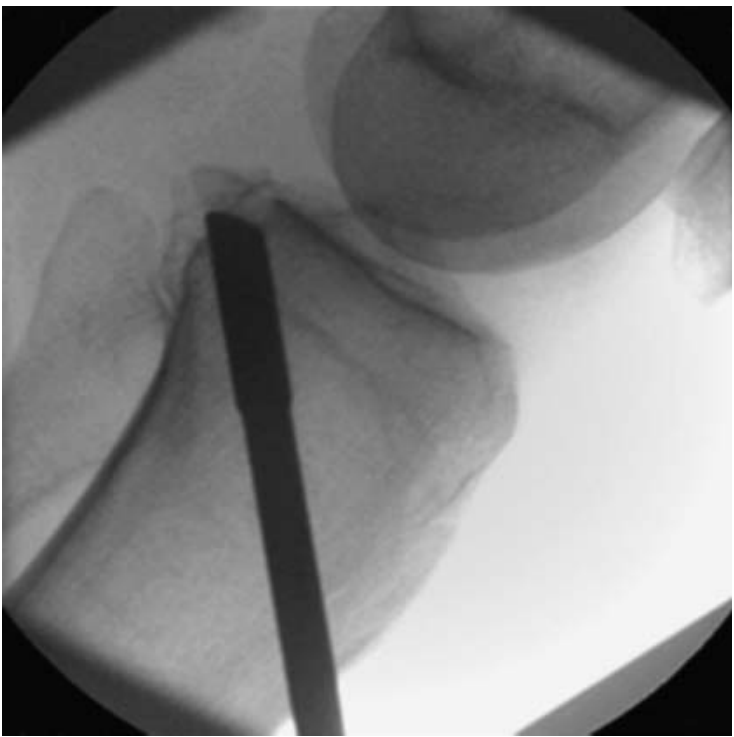

- Abb. 3 Reposition der Fraktur mit dem kanülerten, angeschrägtem Stößel (Arthrex, Naples, USA). 


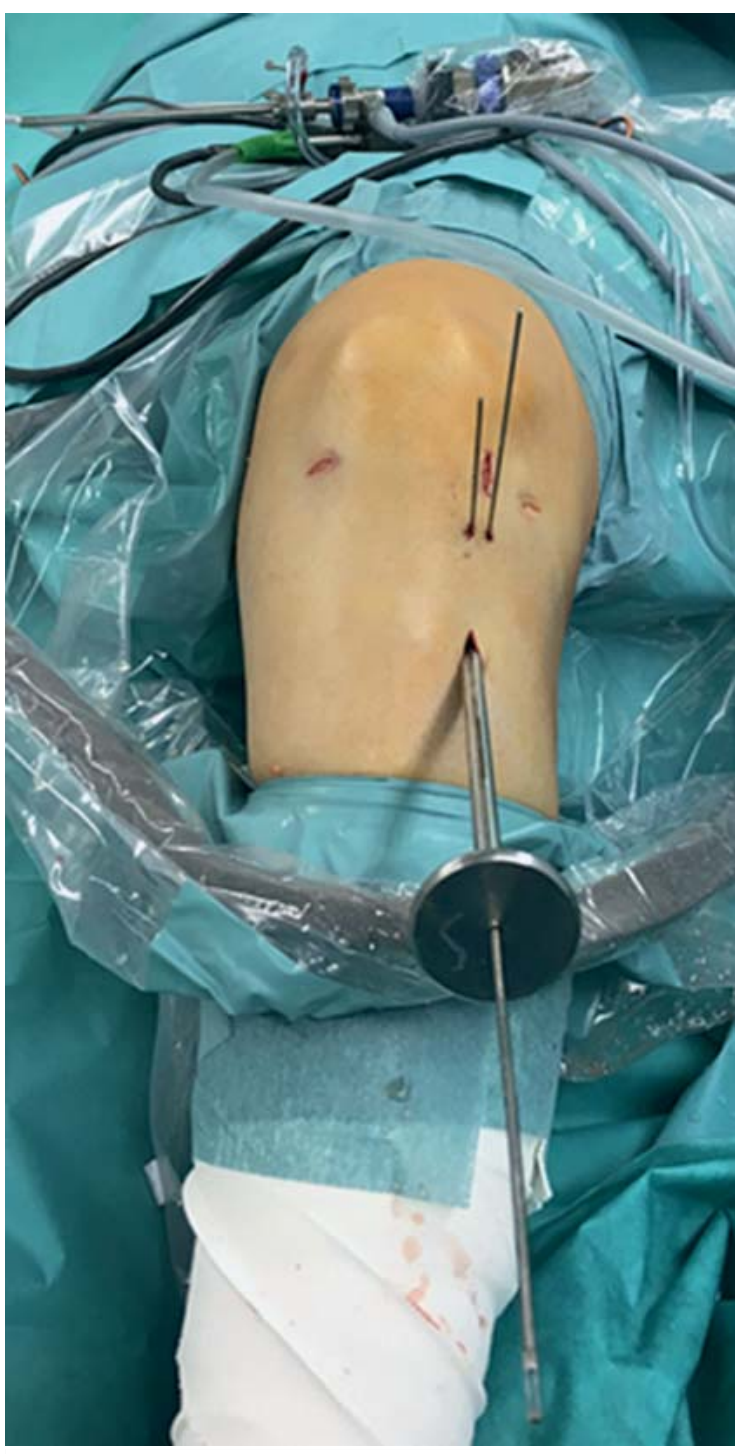

- Abb. 4 Intraoperatives Setting mit perkutanem Einbringen des Repositionsstößels.

über 2-3 kanülierte Schrauben fixiert werden ( $\bullet$ Abb. 47).

Der kanülierte, angeschrägte Stößel ermöglicht eine gezielte, minimalinvasive und stabile Versorgung posterolateraler Kantenfragmente am Tibiakopf. Grundsätzlich kann der gezeigte Stößel für die Reposition nahezu aller Impressions- oder Depressionsfrakturen am Tibiakopf verwendet werden.

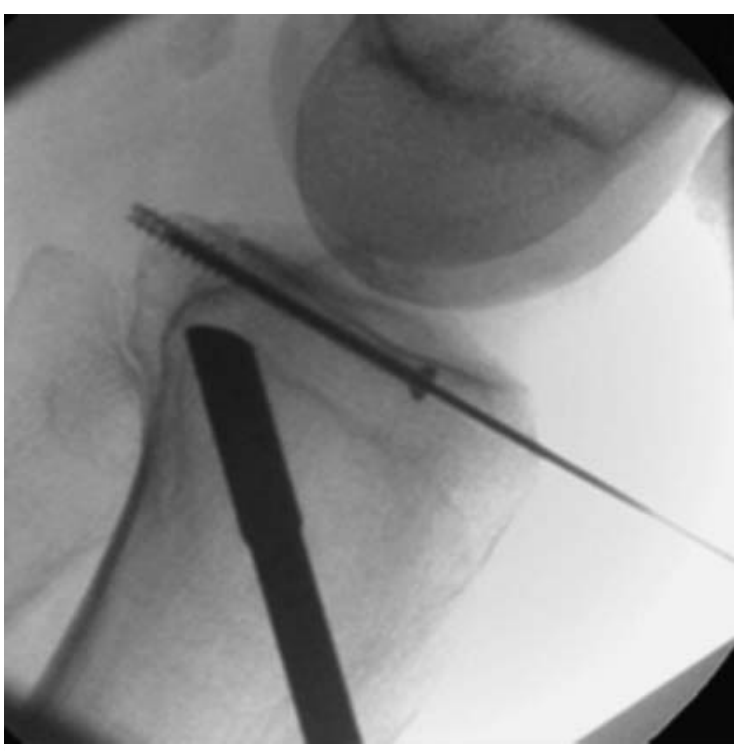

Abb. 5 Stabilisierung der Fraktur im seitlichen Strahlengang mit kanülierten Zugschrauben.

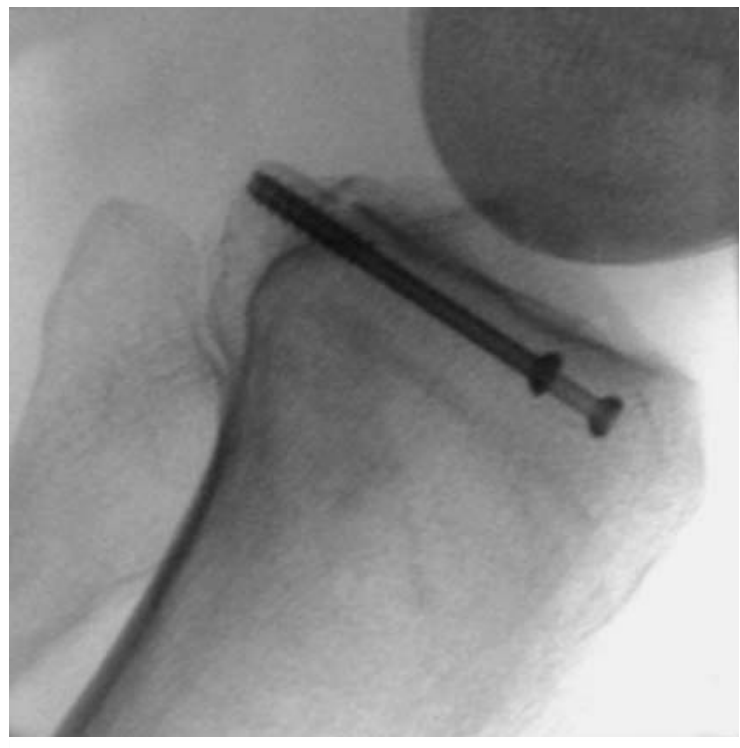

- Abb. 6 Intraoperative Kontrolle der Schraubenlage. 


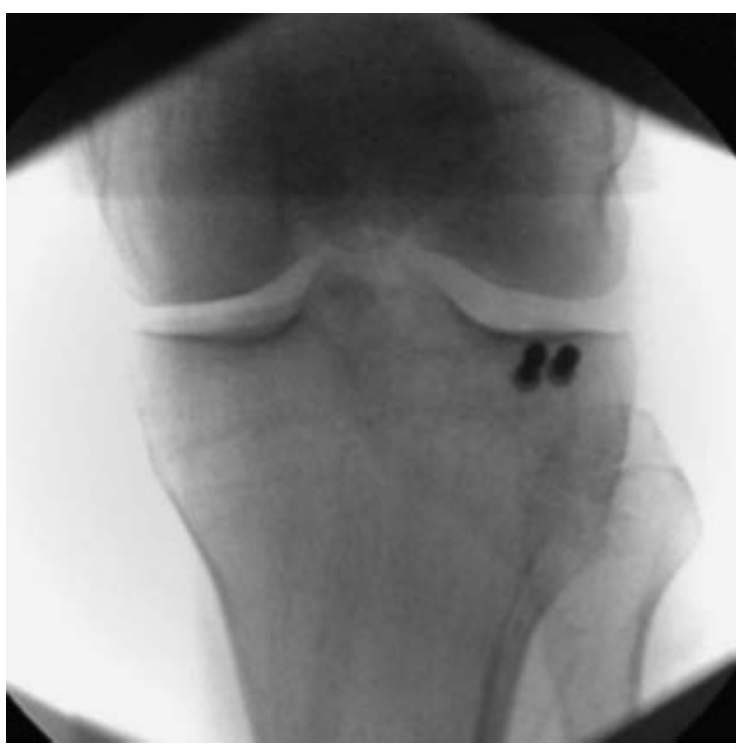

Abb. 7 a.-p.-Ansicht nach Reposition und Schraubenosteosynthese.

\section{Autorinnen/Autoren}

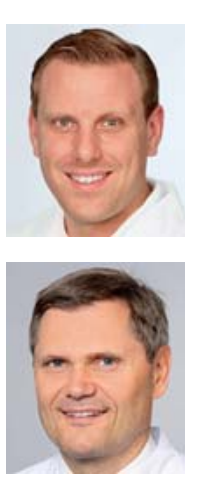

Klinik und Poliklinik für Unfallchirurgie, Handchirurgie und Wiederherstellungschirurgie, Universitätsklinikum Hamburg-Eppendorf UKE

\section{Univ.-Prof. Dr. Karl-Heinz Frosch}

Klinik und Poliklinik für Unfallchirurgie, Handchirurgie und Wiederherstellungschirurgie, Universitätsklinikum Hamburg-Eppendorf UKE

\section{Univ.-Prof. Dr. Karl-Heinz Frosch}

Klinik und Poliklinik für Unfallchirurgie, Handchirurgie und Wiederherstellungschirurgie

Universitätsklinikum Hamburg-Eppendorf UKE

Martinistraße 52

20246 Hamburg

Tel.: 0 40/7410-53459

Fax: 040/74 10-54569

unfallchirurgie@uke.de

\section{Literatur}

[1] Frosch $\mathrm{KH}$, Balcarek P, Walde T et al. A new posterolateral approach without fibula osteotomy for the treatment of tibial plateau fractures. J Orthop Trauma 2010; 24: 515-520

[2] Krause M, Preiss A, Muller G et al. Intra-articular tibial plateau fracture characteristics according to the "ten segment classification". Injury 2016; 47: 2551-2557

[3] Meulenkamp B, Martin R, Desy NM et al. Incidence, risk factors, and location of articular malreductions of the tibial plateau. J Orthop Trauma 2017; 31: 146-150

[4] van den Berg J, Reul M, Nunes Cardozo M et al. Functional outcome of intra-articular tibial plateau fractures: the impact of posterior column fractures. Int Orthop 2017; 41: 1865-1873

\section{Bibliografie}

DOI https://doi.org/10.1055/a-0677-3651

OP-JOURNAL 2019; 35: 63-65 @ Georg Thieme Verlag KG Stuttgart · New York ISSN 0178-1715 